\title{
Ape duos and trios: Spontaneous cooperation with free partner choice in chimpanzees
}

The purpose of the present study was to push the boundaries of cooperation among captive chimpanzees (Pan troglodytes). There has been doubt about the level of cooperation that chimpanzees are able to spontaneously achieve or understand. Would they, without any pretraining or restrictions in partner choice, be able to develop successful joint action? And would they be able to extend cooperation to more than two partners, as they do in nature? Chimpanzees were given a chance to cooperate with multiple partners of their own choosing. All members of the group ( $\mathrm{N}=11)$ had simultaneous access to an apparatus that required two (dyadic condition) or three (triadic condition) individuals to pull in a tray baited with food. Without any training, the chimpanzees spontaneously solved the task a total of 3,565 times in both dyadic and triadic combinations. Their success rate and efficiency increased over time, whereas the amount of pulling in the absence of a partner decreased, demonstrating that they had learned the task contingencies. They preferentially approached the apparatus when kin or nonkin of similar rank were present, showing a preference for socially tolerant partners. The forced partner combinations typical of cooperation experiments cannot reveal these abilities, which demonstrate that in the midst of a complex social environment, chimpanzees spontaneously initiate and maintain a high level of cooperative behavior. 
1 Ape duos and trios: Spontaneous cooperation with free partner choice in 2 chimpanzees

3 Malini Suchak $^{1,2,3^{*}}$, Timothy M. Eppley ${ }^{1}$, Matthew W. Campbell ${ }^{1} \&$ Frans B.M. de Waal ${ }^{1,2}$

$4 \quad{ }^{1}$ Living Links, Yerkes National Primate Research Center, Lawrenceville, GA 30043

$5 \quad{ }^{2}$ Department of Psychology, Emory University, Atlanta, GA 30322

$6 \quad{ }^{3}$ Department of Animal Behavior, Ecology \& Conservation, Canisius College, Buffalo, NY

$7 \quad 14201$

8 *To whom correspondence should be addressed: Malini Suchak, suchakm@canisius.edu, 2001

9 Main St, Buffalo, NY 14208, 1-716-888-2720. 


\section{Abstract}

11 The purpose of the present study was to push the boundaries of cooperation among captive

12 chimpanzees (Pan troglodytes). There has been doubt about the level of cooperation that

13 chimpanzees are able to spontaneously achieve or understand. Would they, without any pre-

14 training or restrictions in partner choice, be able to develop successful joint action? And would

15 they be able to extend cooperation to more than two partners, as they do in nature? Chimpanzees

16 were given a chance to cooperate with multiple partners of their own choosing. All members of

17 the group $(\mathrm{N}=11)$ had simultaneous access to an apparatus that required two (dyadic condition) or

18 three (triadic condition) individuals to pull in a tray baited with food. Without any training, the

19 chimpanzees spontaneously solved the task a total of 3,565 times in both dyadic and triadic

20 combinations. Their success rate and efficiency increased over time, whereas the amount of

21 pulling in the absence of a partner decreased, demonstrating that they had learned the task

22 contingencies. They preferentially approached the apparatus when kin or nonkin of similar rank

23 were present, showing a preference for socially tolerant partners. The forced partner

24 combinations typical of cooperation experiments cannot reveal these abilities, which demonstrate

25 that in the midst of a complex social environment, chimpanzees spontaneously initiate and

26 maintain a high level of cooperative behavior.

27 Key Words: Cooperation, Chimpanzee, Pan troglodytes, Partner Choice, Tolerance 


\section{Introduction}

Cooperation, joint action by two or more individuals to achieve a goal, is often regarded as less puzzling than altruistic behavior, in which one individual benefits another at a cost to himself or herself. While this may be true in an evolutionary sense, on a proximate level, cooperation often consists of a series of potentially complex decisions involving a choice of partners. When multiple potential partners are available, an individual faces many questions: Whom to cooperate with? Has that individual been a good partner in the past? How much to invest in this partner and what to expect in return? Will cooperation yield more benefits than solitary action?

These questions highlight the complexity of cooperative behavior, and yet for such a seemingly complex phenomenon it is surprisingly ubiquitous across a wide variety of taxa (Dugatkin 1997; Gadakar 2006). This paradox has inspired research examining the emergence and maintenance of cooperative behavior at both the ultimate and proximate levels of explanation. In particular, cooperation among nonhuman primates has attracted considerable research because of the evolutionary implications that such research has for human behavior and the ubiquity of cooperation among wild primates, including coalition formation, food sharing, group hunting, and territorial defense (Mueller \& Mitani 2005; Mitani 2006; de Waal \& Suchak 2010). Nevertheless, we know little about the proximate mechanisms of primate cooperation. Do primates coordinate their behavior in space and time? Do they keep track of favors given and received? Do they understand whether and how their partners contribute to successful outcomes?

Or do they just simultaneously pursue the same goal? Conceivably, the appearance of cooperation could be created by parties focused entirely on their own individual gain (Stanford 1998). Given the ambiguity of the field data, experimental studies of cooperation have focused on elucidating the underlying cognitive and social mechanisms. 
53 pairs. Coordinated lever-pressing studies required two monkeys to simultaneously press levers or

54 pull handles to receive food (Chalmeau 1994; Chalmeau et al. 1997; Visalberghi et al. 2000).

55 These studies mostly demonstrated conditioned responses without an understanding of the

56 contingencies: both individuals continually and rapidly pressed the lever and occasionally

57 succeeded by coincidence (Visalberghi et al. 2000). In contrast, when two individuals were

58 required to pull in a weighted tray too heavy for one individual, several primate species

59 demonstrated an ability to coordinate pulling rather than instrumental conditioning (de Waal \&

60 Berger 2000; Crawford 1937; Cronin et al. 2005; Mendres \& de Waal 2000). Subjects

61 demonstrated an understanding of the need for a partner: a juvenile chimpanzee (Pan troglodytes)

62 would recruit a partner through gestures (Crawford 1937), cotton-top tamarins (Saguinus

63 oedipus) pulled more when a partner was present than when a partner was absent (Cronin et al.

64 2005), and brown capuchin monkeys (Cebus apella) were unsuccessful if they could not see each

65 other, suggesting visual coordination (Mendres \& de Waal 2000). The high degree of success in

66 these tasks, as compared to lever-pressing studies has been attributed to the intuitive nature of

67 pulling in a tray baited with food (Mendres \& de Waal 2000). In weighted tray tasks, individuals

68 can clearly see the results of their actions and the role of their partner.

69 Similarly, primates are quite successful at cooperative string-pulling tasks, which require

70 two individuals to simultaneously pull a loose string to bring in a tray of food (Hirata 2003, as

71 cited in Hirata \& Fuwa 2007). These studies differ from the weighted tray and lever-pressing

72 studies in that responding before a partner causes the string to release, rendering both subjects

73 unable to solve the task. The critical test is a delay test in which one individual arrives at the

74 apparatus before the other. Understanding of the cooperative nature of the task requires this

75 individual to wait for the second individual before pulling. This is precisely what has been found

76 in several mammal species (chimpanzees: Hirata \& Fuwa, 2007; Melis et al 2006a; hyenas 
77 (Crocuta crocuta): Drea \& Carter 2009; elephants (Elephas maximus): Plotnik et al. 2011). Not

78 all species pass this critical test: two bird species able to pull simultaneously, failed to wait for

79 their partner in the delay task (rooks (Corvus frugilegus): Seed et al. 2008; parrots (Psittacus

80 erithacus): Péron et al. 2011).

81 A limitation of most experimental studies is the elimination of partner choice. This holds

82 for virtually all primate studies (e.g. Crawford 1937; Hirata \& Fuwa 2007) but also for

83 experiments on non-primates, such as elephants or birds (Seed et al. 2008; Plonik et al. 2011;

84 Peron et al. 2011). An exception is the work by Melis et al. (2006b; 2008), which allowed a

85 choice between two potential partners and demonstrated that chimpanzees differentiate between

86 them based on social tolerance and past success. Yet, the partner choice presented in these

87 experiments was still greatly limited compared to the options within an open group setting.

88 Studies that have allowed open partner choice have generally not found high degrees of

89 cooperation (Burton 1997; Chalmeau 1994; Chalmeau \& Gallo 1996; Chalmeau et al. 1997; Fady

90 1972; Petit et al 1992). In fact, the only species to succeed on such a task were Tonkean

91 macaques (Macaca tonkeana; Petit et al. 1992). Although chimpanzees and capuchins succeed at

92 dyadic pulling tasks (Melis et al. 2006a; Mendres \& de Waal 2000), and are known for

93 cooperative behavior in nature (reviewed by de Waal \& Suchak 2010), both species failed to

94 establish cooperation in studies offering free partner choice (Chalmeau 1994; Chalmeau \& Gallo

95 1996; Chalmeau et al. 1997). Two possibilities are raised by these results: first, tolerance may be

96 so constrained in the group setting that it prevents cooperation without experimenter interference.

97 Although this idea is supported by the fact that the highly tolerant Tonkean macaques are the only

98 species that succeeded at the task (Petit et al 1992), if true partner choice is available, individuals

99 should simply be able to avoid intolerant partners. A second possibility is that the design of the

100 tasks, with one small, highly monopolizable food source as the reward contributed to a contest 
101

102

104

105

106

107

108

109

110

111

112

113

114

competition in which there was not an overall net benefit for all participants (e.g. Chalmeau 1994, Chalmeau et al 1997).

In the current study we allow all individuals access to the apparatus while choosing their own partners. To find out which partners chimpanzees prefer to cooperate with, we carried out experiments in a large outdoor enclosure with the entire group present. All chimpanzees could potentially participate in the cooperative task. We explored several determinants of partner choice. If social closeness were the primary constraint on partner choice (Melis et al. 2006a), then chimpanzees should work mostly with kin or nonkin affiliates. Closeness in dominance rank may also play a role in cooperation, since closely ranked individuals have similar abilities and needs and may be in the best position to benefit each other (de Waal \& Luttrell 1986; Mueller \& Mitani 2005). Cercopithecine monkeys and chimpanzees who are close in rank tend to interact more than those at greater rank distances (Silk 1982; de Waal 1991; Mueller \& Mitani 2005). Rank distance may also play an important role when there is competition for resources (e.g. de Waal 1986), as individuals who are close in rank tend to be more tolerant of each other's presence near a clumped resource. If the presence of the group in the current cooperation task engenders competitive tendencies, then rank distance is expected to affect partner choice. Finally, an alternative hypothesis unrelated to social relationships is that chimpanzees will preferentially choose to work with those with whom they have enjoyed previous successes (Melis et al. 2006b).

In addition to allowing partner choice, the current study further deviates from the previous work by testing both dyadic and triadic cooperation. In nature, chimpanzee cooperation often requires working with more than one other individual, including coalitions, group hunting and territorial defense (Mueller \& Mitani 2005). In Kibale National Park in Uganda, for example, male chimpanzees hunt in groups and an increasing number of hunters leads to increased success even though this effect plateaus at six individuals (Mitani \& Watts 1999). 
126 due to the nature of their fission-fusion society, relatively little is known about the dynamics of

127 female-female cooperation. The current study allows us to explore how female chimpanzees

128 choose and switch partners when freed from their natural ecological constraints. Since females of

129 the closely related bonobo are highly cooperative (Furuichi, 2011; Hare et al., 2007), examining

130 cooperation in female chimpanzees can help shed light on the evolution of cooperative

131 tendencies.

132 Methods

\section{Subjects and Housing}

134 Subjects were 11 chimpanzees (1 male, 10 females, Table 1) kept in a large outdoor 135 enclosure at the Field Station of the Yerkes National Primate Research Center (YNPRC). The 136 group's $711 \mathrm{~m}^{2}$ enclosure contained a large climbing structure and several enrichment items 137 (barrels, tires, etc.) and was adjacent to indoor sleeping quarters. Testing occurred in the outdoor 138 enclosure with the entire group present and did not require separating individuals from the group.

139 During testing, subjects had access to the indoor sleeping quarters. Chimpanzees were fed two 140 daily meals consisting of fruits, vegetables and grains at approximately $8 \mathrm{~h} 30$ and $15 \mathrm{~h} 00$ and had 141 access to water and primate chow ad libitum. All food used in this study was supplemental to the 142 chimpanzees daily intake and at no time was food or water restricted. The chimpanzees were not 143 distressed and were free to stop participating at any time. All procedures were approved by 144 Emory University's Institutional Animal Care and Use Committee (IACUC), protocol \#YER145 2000180-53114GA. The Yerkes National Primate Research Center is furthermore fully 146 accredited by the Association for Assessment and Accreditation of Laboratory Animal Care 147 (AAALAC). 
151 (in the triadic condition) remove a barrier in order for another chimpanzee to simultaneously pull

152 in a tray baited with food (Figure 1). The second barrier and the corresponding pull bar were only

153 present in triadic tasks, providing a clear cue to the chimpanzees that a third individual was

154 needed. Once the tray was pulled in all the way (approximately $30 \mathrm{~cm}$ ) food rewards dropped into

155 a funnel, which delivered them directly to each of the chimpanzees that solved the task. The rods

156 to pull down the barriers and pull in the tray were sufficiently far apart $(\sim 1.6 \mathrm{~m})$ so that one

157 individual could not participate in both roles at the same time. If a barrier rod was pulled and

158 released before the tray was pulled in, the barrier rose back into place. Hence, a lone individual

159 could not remove the barrier and pull in the tray; simultaneous action by multiple chimpanzees

160 was required. Food rewards (one grape, two raisins, a small slice of sweet potato or a small slice

161 of banana) varied randomly from trial to trial to maintain the chimpanzees' interest; for each trial

162 all chimpanzees received the same reward. These were not rewards that the chimpanzees received

163 outside the experimental context, thus further increasing their motivation to participate in the 164 task.

\section{FIGURE 1 ABOUT HERE}

\section{Procedures}

167 Test Sessions. A trial began when the tray was baited with food. Following successful trials, the

168 experimenter waited for the chimpanzees to release the rods and then reset the tray back in the

169 starting position and re-baited the tray. The tray was re-baited as long as the chimpanzees present

170 were not pulling; they did not need to leave and re-approach between trials. If the chimpanzees

171 did not solve the task within 5 minutes, the trial was considered a failure, the food removed, and a

172 one-minute time out commenced prior to re-baiting. Each session lasted 1 hour and consisted of

173 as many trials as could be accomplished in that time period. Only one session was run per day 
174 and only 2-3 sessions were run per week to maintain a high degree of motivation. Sessions were 175 run from May 2011 through February 2012.

176 Test Phases.

177 Phase 1a: Dyadic cooperation acquisition. There were 28 dyadic cooperation tests, which 178 required two chimpanzees to work together to pull in the tray (one to hold down the barrier 179 and a second to pull in the tray). As there was no training, and none of the chimpanzees had 180 participated in cooperative pulling tasks before, we waited for a significant majority ( 9 out of

181 chimpanzees, binomial test $\mathrm{p}=0.02$ ) of the chimpanzees to reach at least 20 successes 182 before moving onto the next phase.

183 Phase 1b: Triadic cooperation acquisition. Following Phase 1a a second barrier was added so 184 that three chimpanzees were required to solve the task. There were 28 triadic cooperation 185 tests, to allow for direct comparison to the acquisition of dyadic cooperation.

$186 \quad$ Phase 2: Alternating, proficiency tests. In order to see if proficiency and experience with 187 triadic cooperation influenced partner choice, we began alternating dyadic and triadic 188 sessions. There were 38 alternating sessions, or 19 of each dyadic and triadic.

\section{Behavioral Coding}

190 Each trial was videotaped from two angles (an overview from above, taken from an

191 observation tower, and a front view) using HD digital video cameras. Additionally, one

192 experimenter had a digital voice recorder to record a narrative of any social interactions that

193 occurred during testing. Success or failure of each trial, which chimpanzees solved the task, and

194 which chimpanzees received rewards were recorded in-person and later confirmed from video. 


\section{Analyses}

215 Understanding of the task. To test whether the chimpanzees learned about the need for and role of

216 the partner, we compared behavior during the acquisition phases to the proficiency phase. Within-

217 subjects mixed measures ANOVAs were run to compare differences between phases and partner

218 conditions (dyadic and triadic) for latency, efficiency, and pulling. Latency and efficiency

\section{FIGURE 2 ABOUT HERE}


219 (number of pulls to success) were both measured from the time the succeeding pair or triad

220 arrived at the apparatus until the time of success, when they obtained the food. We compared the

221 pulling rate (pulls per minute) of each individual when the correct number of partners was at the

222 bar "ready" to pull versus when there were not enough partners present. This is a very strict

223 criterion as chimpanzees who momentarily stepped away from the apparatus or were approaching

224 but not yet within reach of the bar were not considered to be "present." In the dyadic condition

225 we compared the pulling rate when a partner was present versus absent and in the triadic

226 condition we compared the pulling rate when all three partners were in place to when only two or

227 one partners were in place. We compared these rates between the acquisition and proficiency

228 phases to check for developing understanding of the need for a partner over time. All pulls, even

229 those that occurred in the absence of success were included in this analysis. All acquisition

230 analyses were run using SPSS Statistics 20.0 (IBM, inc.).

231 Partner Choice. We explored which chimpanzees chose to approach the apparatus when other

232 chimpanzees were already there as potential partners. When a chimpanzee was present at the

233 apparatus, he or she had 10 potential partners that could approach. If another individual

234 approached, they were scored as a 1, whereas individuals who did not approach were scored as 0.

235 This was done by session, so if a chimpanzee was never at the apparatus during a given session,

236 they were excluded from the analysis for that session (since they had to be at the apparatus in

237 order for someone to approach to work with them). All triads were broken down into their

238 corresponding dyads for the purpose of analysis. Partner choice analyses are limited to the

239 proficiency phase only as we could confirm at that point the chimpanzees knew they needed a

240 partner.

241 To determine which factors influenced partner choice, we ran a generalized linear mixed

242 model (GLMM), with approach as a binomial dependent variable. Kinship, long-term affiliation, 
243 rank distance between the two individuals, recent success (total number of successes for that pair

244 during the current phase) and relative past success (the percentage of that individual's success

245 from the previous phases with that partner) were included in various combinations as fixed terms.

246 The advantage to using two different measures of past success is that if past success is the key to

247 partner choice, we can determine whether the chimpanzees gauge success based on cumulative

248 rewards obtained (which could result in partner choice by reinforcement) or if they judge partners

249 based on their relative effectiveness at the task (which would reflect a more sophisticated

250 evaluation of available partners). Models examined each fixed effect independently as well as

251 interactions between the effects. None of the fixed effects were correlated with each other. We

252 also ran a full model which contained all of the fixed effects and a null model that contained only

253 the random effects for all phases. Dyadic and triadic sessions were analyzed separately and in

254 total we examined 14 models for each. Identity of the chimpanzee already present at the

255 apparatus, identity of the chimpanzee that approached, and session were included as random

256 effect to control for repeated sampling, frequency of presence at the apparatus, frequency of

257 approach, and interdependence between dyads. We used an ANOVA to determine which model

258 had the most explanatory power by comparing the Akaike's information criterion (AIC) for all of

259 the possible models. Once the best model was identified, we used a Markov chain Monte Carlo

260 simulation of 10,000 interactions to obtain significance values. All partner choice analyses were

261 run using R statistical software (2012), with the lme4 package.

\section{Results}

263 Understanding of the task

264 For both dyadic and triadic tests, at least four different chimpanzees spontaneously solved

265 the task within the first 2 hours of exposure without any training. Overall, 10 of the 11

266 chimpanzees solved the task at least once during both the dyadic and triadic tests for a total of

2672,462 dyadic successes and 1,103 triadic successes. By the end of the acquisition phase, the 
average chimpanzee had succeeded 139 times at the dyadic task and 99 times at the triadic task.

269 This increased to an average success of 447 times at the dyadic task and 301 times at the triadic

270 task by the end of the proficiency phase. The total number of successes by each individual across

271 the entire study is displayed in Figure 3. One female, Mai, failed to solve the task in either dyadic

272 or triadic tests and in fact ceased making pulling attempts before the proficiency phase. Since her

273 overall pulling rates were more than two standard deviations below the group mean, she was

274 eliminated from the analysis.

275

FIGURE 3 ABOUT HERE

276

Latency to success was significantly lower in the proficiency phase than in the acquisition

phase (Mixed Measures ANOVA: $F_{1,7}=21.29, P=0.002$ ). Similarly, extraneous pulling, i.e. pulls

278

that did not lead to success, dropped significantly from the acquisition to the proficiency phase.

279 The chimpanzees succeeded with significantly fewer pulls per success (e.g. higher efficiency)

280 during the proficiency phase (Mixed Measures ANOVA: $F_{1,7}=16.83, P=0.005$, Figure 4). For

281 both of these measures there was no significant difference between dyadic and triadic tests

282 (latency: $F_{1,7}=0.11, P=0.75$; extra pulling: $F_{1,7}=0.18, P=0.68$ ), demonstrating increased

283 effectiveness of cooperation regardless of how many chimpanzees were needed for the task.

284 There were, however significant interactions (latency: $F_{1,7}=13.95, p=0.007$; extra pulling: $F_{1,7}$

$285=10.37, \mathrm{p}=0.015)$, demonstrating a larger change from dyadic acquisition to proficiency than

286 from triadic acquisition to proficiency. It is important to note, however, that these phases for

287 dyads were separated in time by the triadic acquisition phase, whereas the proficiency phase for

288 triads immediately followed the triadic acquisition phase.

FIGURE 4 ABOUT HERE

290

In order to assess whether the chimpanzees developed an understanding of the need for a

291 partner, we compared pulling rates when the correct number of individuals were present and

292 sitting ready at the bars to pull to when an insufficient number was present. In the dyadic test 
293 sessions there was a significant effect of partner presence; chimpanzees pulled more when a

294 partner was at the other bar then when no partner was present (Mixed Measures ANOVA: $F_{1,9}=$

$29539.53, P<0.001$; Figure 4). There was also a significant phase by partner presence interaction:

296 the ratio of pulls when a partner was present as compared to pulls when a partner was absent was

297 greater in the proficiency phase than in the acquisition phase $\left(F_{1,9}=14.11, P=0.005\right)$. Finally,

298 there was an overall effect of phase, such that individuals had higher overall pulling rates in the

299 proficiency phase than in the acquisition phase, however this increase was primarily observed

300 when a partner was present $\left(F_{1,9}=9.76, P=0.01\right)$.

301

FIGURE 4 ABOUT HERE

302

In triadic tests, the trends were similar but less pronounced. There was a main effect of

303 partner presence: when two other partners were present the chimpanzees pulled more than when

304 there was only one or zero partners present $\left(F_{1.12,10.99}=11.62, p=0.006\right.$, Greenhouse-Geisser

305 corrected due to lack of sphericity). Unlike dyadic tests, however, the phase by number of

306 partners interaction was no longer significant $\left(F_{1.07,9.59}=0.962, p=0.36\right.$, Greenhouse-Geisser

307 corrected due to lack of sphericity) and there was no longer a main effect of phase $\left(F_{1,9}=2.49, p\right.$ $308=0.15)$.

309 Most of the chimpanzees spontaneously developed a bias for a particular position at the

310 apparatus. In the dyadic task, three chimpanzees had significantly more success (as determined

311 by a binomial test $P<0.05$ ) at the barrier than the tray position, two chimpanzees had no

312 preference and five chimpanzees had significantly more success at the tray than the barrier

313 position. In the triadic condition, there was more skew towards barrier preference, likely due to

314 the fact that there were two barrier positions available and only one tray position.

\section{Partner choice}


317 combinations among all of the adults who solved the task $(N=10)$. In total, 29 unique dyads and

31832 unique triads manifested actual cooperation in the experiments.

319 Applying GLMM, we examined factors that might influence whether or not one

320 individual approaches another already present at the apparatus. Across both dyadic and triadic

321 tests, there was a significant influence of the tested random effects (intercept; dyadic: $Z=-4.30$,

$322 P<0.001$; triadic: $Z=-5.11, P<0.001$; random effects included identity of the approacher and

323 individual already there and test session number). Given the high variability of individual

324 participation in the task, it is not surprising that most of the variance of the random effects comes

325 from individual identities rather than the session number (Table 2).

326 Once individual identity was controlled for, the same model had the best fit for both

327 dyadic and triadic sessions. It included kinship, affiliation as well as the interaction of these

328 factors with rank distance (dyadic: $\mathrm{AIC}=601.44, \chi^{2}=9.68, \mathrm{df}=0, P<0.001$; triadic: AIC $=$

329 1199.22, $\chi^{2}=4.12, \mathrm{df}=0, P<0.001$; Table 2). The interaction between kinship and rank

330 distance was significant (dyadic sessions: $Z=3.80, P<0.001$; triadic sessions: $Z=2.67, P=$

331 0.007), reflecting the reluctance of chimpanzees to approach individuals much higher ranking

332 than themselves, unless these individuals were relatives. Additionally, for the dyadic sessions,

333 there were main effects of kinship $(Z=-2.07, P=0.04)$ and rank distance $(Z=-3.86, P<0.001)$

334 but these effects were not found not in triadic sessions (kinship: $Z=-1.05, P=0.30$; rank

335 distance: $Z=-.58, P=0.56)$. Affiliation and the interaction between affiliation and rank distance

336 were not significant in either the dyadic or triadic sessions. Since the best fit model did not

337 include any measure of past success, we examined a full model to see if past success was playing

338 any role in approach. There was no significant effect of recent success or all past success in either

339 the dyadic (recent success: $Z=0.73, \mathrm{P}=0.46$; all past success: $Z=-1.50, \mathrm{P}=0.13$ ) or triadic

340 (recent success: $Z=1.74, \mathrm{P}=0.08$; all past success: $Z=0.35, \mathrm{P}=0.72$ ) proficiency phase. 
343 approach each other. There was no overall effect of rank on task performance, i.e. individuals of

344 high rank did not have more successes than individuals of lower rank (Spearmans' rank

345 correlation between individual rank and number of successes; $r_{s}=0.07, N=10$ individuals, $P=$ 346 0.44).

347 Finally, approaches tended to be reciprocal — that is, the more frequently individual A 348 approached $\mathrm{B}$ at the apparatus, the more frequently B approached A (dyadic: $r_{s}=0.42, N=90, P$ $349<0.001$; triadic: $\left.r_{s}=0.56, N=90, P<0.001\right)$. Note that the $\mathrm{p}$-values reported here are exact two350 tailed p-values obtained from 10,000 random permutations so as to address interdependence 351 between dyads.

\section{Discussion}

Without any specific training, the chimpanzees in this study spontaneously solved the cooperation task and were extremely successful under both dyadic and triadic conditions. The high success rate, with a total of 3,565 completed cooperative acts (an average of 38 per one hour test session), confirms observations of cooperation in nature: chimpanzees are capable of cooperating in more complex open environments than typically tested. The chimpanzees were

358 clearly highly motivated to participate in the task. Since the number of successes during the proficiency phase was higher in both the dyadic and triadic conditions, it is unlikely the

360 chimpanzees habituated to the task. Furthermore, the task was run over 10-month period with an 361 average of 2-3 sessions per week, to prevent both habituation and a loss of motivation from too 362 frequent testing. 
364 studies the chimpanzees required extensive training (Crawford 1937), or had been individually

365 familiarized with the apparatus before any cooperative testing (Melis et al. 2006a; 2006b). In the

366 only previous study without pre-training, 5 out of the 6 chimpanzees showed no understanding of

367 the task and were just as likely to pull when a partner was present versus absent (Chalmeau

368 1994). In the current study, the chimpanzees had no experience with a pulling apparatus of any

369 kind prior to the dyadic acquisition phase. However, it might be argued that the dyadic

370 acquisition phase served as pre-training for the triadic phase. If so, we would expect to see high

371 rates of pulling when only one other partner was present in the triadic phase of testing. This was

372 not the case, however: the chimpanzees pulled the most when both partners were present, less

373 when one partner was present and the least was when no other partner was present. The low

374 frequency of pulling when an insufficient number of partners were present demonstrates an

375 understanding of the triadic nature of the task, which manifested itself right at the beginning of

376 the triadic acquisition phase.

377 Despite the chimpanzees' demonstrable sensitivity to partner presence in both the dyadic

378 and triadic phase, pulling in the absence of a needed partner never fully disappeared. Incomplete

379 extinction of such pulling was probably due to continuing conditioning effects as well as the

380 conservative measure of partner presence employed: the partner had to be at the bar, ready to

381 pull. Therefore, any pulls made as a partner was approaching or nearby were counted as pulling

382 when a partner was "not ready." It is possible that the chimpanzees viewed a partner approach as

383 a signal to start the task. Moreover, pulling was an extremely low cost behavior. The energy

384 expended on pulling might simply not have been great enough to deter extraneous pulling.

385 One of the surprises of this study was the high level of success without any pre-training.

386 Previous work has shown that more intuitive tasks, where individuals are pulling food towards

387 them (e.g. Mendres \& de Waal 2000), are learned faster and showed greater understanding than 
non-intuitive tasks where pulling is not mechanically connected to food delivery. Thus primates

389 participating in weighted tray or string-pulling tasks (Hirata \& Fuwa, 2007; Melis et al 2006ab;

390 Mendres \& de Waal 2000) have had more success than those participating in lever-pressing

391 (Chalmeau et al. 1997, Visalberghi et al. 2000). One exception to this is Crawford's (1937)

392 original weighted tray task, which required extensive training. However, Crawford's chimpanzees

393 were juveniles and in later experiments (including the current study) the participants were all

394 adults. Additionally, the chimpanzees in Crawford's study did show an understanding of the

395 partner's role: one chimpanzee would recruit the other to help him. Since the chimpanzees in the

396 current study could clearly see the mechanical results of their actions and how their actions

397 resulted in food delivery it is not surprising they developed an understanding of the task.

398 Another unexpected finding was how highly successful the apes were despite the group

399 setting in which they were operating. The potential for competition and free-loading did not seem

400 to deter them. Previous studies have demonstrated obstacles to cooperation under free choice

401 conditions due to a lack of inter-individual tolerance (Burton 1997; Chalmeau 1994; Chalmeau \&

402 Gallo 1996; Chalmeau et al. 1997; Fady 1972; Petit et al 1992). However, in all of these studies

403 the reward for cooperation was a single monopolizable food reward. For most participants in the

404 task, there was no net gain. In the current study, in contrast, each individual received their own

405 reward, resulting in a net gain for all participants. Although this design did not allow us to ask

406 how reward division might influence future partner choice, ensuring a net gain for all participants

407 is the essence of mutualism, which allowed us to examine the details of partner choice.

408 Kinship and rank similarity were the best predictors of partner choice: the chimpanzees

409 tended to approach individuals of similar rank to themselves unless the individual at the

410 apparatus was their kin. Interestingly, this did not only apply to low ranking individuals

411 approaching other low ranking individuals; high-ranking individuals also preferred approaching

412 high-ranking individuals. Closeness in rank and kinship probably foster partnerships in which 
413 competition is mitigated (Silk 1982; de Waal \& Luttrell 1986; de Waal 1986). These partnerships

414 are characterized by higher social tolerance than ones with large discrepancies in rank, which

415 often results in the higher-ranking individual forcefully claiming food. Our results are consistent

416 with previous studies that reported higher levels of cooperation between tolerant individuals (de

417 Waal \& Davis 2003; Melis et al. 2006a; Petit et al. 1992). In one previous study of partner choice,

418 the alpha male monopolized the apparatus and rewards, resulting in a lack of interest of the group

419 to approach while he was there (Chalmeau 1994). In the current study, there was a high level of

420 participation by 10 out of the 11 individuals present, acting in a wide variety of partnerships. The

421 alpha male participated, but without excluding others. His most frequent partners were middle- to

422 high-ranking females, i.e. females fairly close to his own rank.

423 Although we only had one male in the group, which limited opportunities to examine

424 male-male cooperation, the number of females that participated in the task allowed us to examine 425 the dynamics of female-female cooperation, which have gone largely understudied. Although

426 wild chimpanzee females rarely cooperate due to ecological constraints, in our study females

427 spontaneously cooperated in both female-female and mixed sex dyads and triads. The females in

428 our study demonstrated the high potential for female-female cooperation and allowed us to

429 investigate the dynamics of female partner choice.

430 Further evidence of the high level of social tolerance between partners is demonstrated by

431 the low rate of agonism observed throughout the study. Agonism was extremely rare, occurring in

432 only about $1 \%$ of all trials. Escalated agonism (e.g. slapping, biting, or grabbing) was rarer still,

433 occurring in only $0.1 \%$ of trials.

434 The emphasis on tolerant partnerships means that the chimpanzees were not choosing the

435 most successful partners available. These results, combined with previous work (Burton 1997;

436 Chalmeau 1994; Chalmeau \& Gallo 1996; Chalmeau et al. 1997; Fady 1972; Petit et al 1992)

437 seem to suggest that while theoretically the chimpanzees should be choose the most successful 
438 individuals to maximize their own gain, there may be social constraints on their ability to display

439 this tendency. Indeed, when social constraints are taken away by limiting partner choice to only

440 two individuals who were socially tolerant, chimpanzees did choose the most successful partners

441 (Melis et al 2006b). From an evolutionary standpoint, social relationships are long-term

442 investments that encompass a variety of interactions (including grooming, agonistic support, sex,

443 play, and food sharing). Cooperation is only one of many different currencies being exchanged in

444 a marketplace. Rather than being "irrational," choosing a tolerant partner may reflect the most

445 economical choice: a safe investment that is likely to lead to equal outcomes for all participants,

446 in the present and in future interactions.

\section{Acknowledgements}

448 We would like to thank Victoria Horner, Darby Proctor, Zanna Clay, Harold Gouzoules, Sarah 449 Brosnan, Monica Capra, and Philippe Rochat for helpful discussions; Julia Watzek for statistical 450 help; the Veterinary and Animal Care staffs at the Yerkes National Primate Research Center for 451 maintaining the health of our research subjects. The YNPRC is fully accredited by the American 452 Association for Accreditation for Laboratory Animal Care (AAALAC).

\section{Funding Statement}

454 This study was supported by Living Links, Emory's PRISM Program (NSF GK12

455 \#DGE0536941), Emory's Dean's Teaching Fellowship program, Emory's FIRST program 456 (NIH/NIGMS(USA) IRACDA grant \#K12GM00680 to M.W.C), the Greater Good Science 457 Center at the University of California-Berkeley, and the base grant of the National Institutes of 458 Health to the YNPRC from the National Center for Research Resources PR51RR165 (currently 459 supported by the Office of Research Infrastructure Programs/ODP51OD11132).

\section{References}

461 Burton, J. J. 1977. Absence de comportement coopératif spontané dans une troupe de Macaca 462 fuscata en presence de pierres appâtées. Primates, 18, 359-366.

463 Chalmeau, R. 1994. Do chimpanzees cooperate in a learning task? Primates, 35, 385-392.

464 Chalmeau, R. \& Gallo, A. 1996. What chimpanzees (Pan troglodytes) learn in a cooperative task. 465 Primates, 37, 39-47.

466 Chalmeau, R., Visalberghi, E. \& Gallo, A. 1997. Capuchin monkeys, Cebus apella fail to 467 understand a cooperative task. Animal Behaviour, 54, 1215-1225. 
468

469

470

471

472

473

474

475

476

477

478

479

480

481

482

483

484

485

486

487

488

489

490

491

492

493

494

495

496

497

498

Crawford, M. 1937. The cooperative solving of problems by young chimpanzees. Comparative Psychology Monographs, 14, 1-88.

Cronin, K.A., Kurlan, A.V. \& Snowdon, C.T. 2005. Cooperative problem solving in a cooperatively breeding primate (Saguinus oedipus). Animal Behaviour, 69, 133-142.

de Waal, F. B. M. 1986. Class structure in a rhesus monkey group: The interplay between dominance and tolerance. Animal Behaviour, 34, 1033-1040.

de Waal, F.B.M. 1989. Food sharing and reciprocal obligations among chimpanzees. Journal of Human Evolution, 18, 438-459.

de Waal, F. B. M. 1991. Rank distance as a central feature of rhesus monkey social organization: a sociometric analysis. Animal Behaviour, 41, 383-395.

de Waal, F.B.M. \& Berger, M.L. 2000. Payment for labour in monkeys. Nature, 404563.

de Waal, F. B. M. \& Davis, J. M. 2003. Capuchin cognitive ecology: cooperation based on expected returns. Neuropsychologia, 41, 221-228.

de Waal F. B. M. \& Luttrell L. M. 1986. The similarity principle underlying social bonding among female rhesus monkeys. Folia Primatologica, 46, 215-234.

de Waal, F. B. M. \& Suchak, M. 2010. Prosocial primates: selfish and unselfish motivations. Philosophical Transactions of the Royal Society B, 365, 2711-2722.

Drea, C. M. \& Carter, A. N. 2009. Cooperative problem solving in a social carnivore. Animal Behaviour, 78, 967-977.

Dugatkin, L. A. 1997. Cooperation Among Animals. Oxford: Oxford University Press.

Everitt, B. 1977. The analysis of contingency tables. London: Chapman \& Hall.

Fady, J. C. 1972. Absence de cooperation de type instrumental en milieu naturel chez Papio papio. Behaviour, 43, 157-164.

Furuichi, T. (2011). Female contributions to the peaceful nature of bonobos society. Evolutionary Anthropology, 20, 131-142.

Gadakar, R. 2006. Survival Strategies: Cooperation and Conflict in Animal Societies. Cambridge, MA: Harvard University Press.

Hare, B., Melis, A.P., Woods, V., Hastings, S., \& Wrangham, R. (2007). Tolerance allows bonobos to outperform chimpanzees on a cooperative task. Current Biology, 17, 619-623.

Hirata, S. \& Fuwa, K. 2007. Chimpanzees (Pan troglodytes) learn to act with other individuals in a cooperative task. Primates, 48, 13-21. 
499

500

501

502

503

504

505

506

507

508

509

510

511

512

513

514

515

516

517

518

519

520

521

522

523

524

525

526

527

528

529

Melis, A. P., Hare, B. \& Tomasello, M. 2006a. Engineering cooperation in chimpanzees: tolerance constraints on cooperation. Animal Behaviour, 72, 275-286.

Melis, A. P., Hare, B. \& Tomasello, M. 2006b. Chimpanzees recruit the best collaborators. Science, 311, 1297-1300.

Melis, A. P., Hare, B. \& Tomasello, M. 2008. Do chimpanzees reciprocate received favours? Animal Behaviour, 76, 951-962.

Mendres, K.A. \& de Waal, F.B.M. 2000. Capuchins do cooperate: the advantage of an intuitive task. Animal Behaviour, 60, 523-529.

Mitani, J.C. 2006. Reciprocal exchange in chimpanzees and other primates. In Cooperation in primates and humans: Evolution and Mechanisms (ed. P.M. Kappeler and C.P. van Schaik), pp. 107-120, Berlin: Springer.

Mitani, J. C. \& Watts, D. P. 1999. Demographic influences on the hunting behavior of chimpanzees. American Journal Physical Anthropology, 109, 439-454.

Muller, M. N. \& Mitani, J. C. 2005. Conflict and cooperation in wild chimpanzees. Advances in the Study of Behavior, 35, 275-331.

Péron, F., Rat-Fischer, L., Lalot, M., Nagle, L., \& Bovet, D. 2011. Cooperative problem solving in African grey parrots (Psittacus erithacus). Animal Cognition, 14, 545-553.

Petit, O., Desportes, C., \& Thierry, B. 1992. Differential probability of "coproduction" in two species of macaque (Macaca tokeana, M. mulatta). Ethology, 90, 107-120.

Plotnik, J. M., Lair, R., Suphachoksahakun, W., de Waal, F. B. M. 2011. Elephants know when they need a helping trunk in a cooperative task. Proceedings of the National Academy of Sciences, 108, 5116-5121.

Seed, A. M., Clayton, N. S. \& Emery, N. J. 2008. Cooperative problem solving in rooks (Corvus frugilegus). Proceedings of the Royal Society B, 275, 1421-1429.

Silk, J.1982. Altruism among female Macaca radiata: explanations and analysis of patterns of grooming and coalition formation, Behaviour 79, 162-188.

Stanford, C. B. 1998 Chimpanzee and Red Colobus: The Ecology of Predator and Prey. Cambridge, MA: Harvard University Press.

Visalberghi, E., Quarantotti, B.P. \& Tranchida, F. 2000. Solving a cooperation task without taking into account the partner's behavior: the case of capuchin monkeys (Cebus apella). Journal of Comparative Psychology, 114, 297-301. 
531 Figure 1. The test apparatus and set-up. (a) Each barrier was connected to a steel rod that

532 extended $20 \mathrm{~cm}$ into the chimpanzee enclosure. Pulling on the rod caused the barrier to drop

533 down via a spring/pulley mechanism. Once the barrier (or barriers in the triadic condition) was

534 pulled down a second individual used a similar rod (also extending $20 \mathrm{~cm}$ into the enclosure) to

535 pull in the whole tray. The only part of the test apparatus that was inside the enclosure were the

536 pull bars, the rest was outside. Note that barrier 2 (on the left) was only present during the triadic

537 sessions. The apparatus was set up exactly the same for dyadic sessions but barrier 2 and the

538 corresponding pull bar were missing. (b) Three chimpanzees participating in the triadic

539 cooperation task.

540 Figure 2. Affiliation sociogram of the chimpanzee group. Linkages between individuals

541 indicates a significant, positive association. The thicker the line, the stronger the association

542 between individuals. The alpha male (Socko) and alpha female (Georgia) are denoted with an $\alpha$.

543 Figure 3. Success by individual chimpanzee. The total number of successes for each

544 chimpanzee across all of the test sessions. All individuals except for MA achieved at least 80

545 successes, with nine chimpanzees achieving over 100.

Figure 4. Number of pulls to success during the acquisition and proficiency phases.

547 Extraneous pulling that did not lead to success decreased between the two phases for both dyadic

548 and triadic partners. There was no significant difference between the dyadic and triadic

549 conditions.

550

Figure 5. Comparison of pulling rates per minute during the acquisition and proficiency

551

552

553

phases for dyadic cooperation. Partner "ready" indicates that a partner was both present at the apparatus in a position to be able to pull on the tray. The pattern was similar for triadic pulling rates.

\section{Table captions}

555 Table 1. Subjects. Subjects are listed in rank order, with age and any maternal kinship relations 556 provided. Rank was determined using pant grunts, a unidirectional submissive signal. Alpha male 557 and female are denoted with an $\alpha$.

558 Table 2. Results of the best fit GLMM during the proficiency phase. Fixed effects in bold had

559 a significant influence on whether or not an individual approached. In both dyadic and triadic

560 sessions, individuals were more likely to approach others close in rank to themselves, unless the

561 potential partner was kin.

562 


\section{Figure 1}

The test apparatus and set-up

(a) Each barrier was connected to a steel rod that extended $20 \mathrm{~cm}$ into the chimpanzee enclosure. Pulling on the rod caused the barrier to drop down via a spring/pulley mechanism. Once the barrier (or barriers in the triadic condition) was pulled down a second individual used a similar rod (also extending $20 \mathrm{~cm}$ into the enclosure) to pull in the whole tray. The only part of the test apparatus that was inside the enclosure were the pull bars, the rest was outside. Note that barrier 2 (on the left) was only present during the triadic sessions. The apparatus was set up exactly the same for dyadic sessions but barrier 2 and the corresponding pull bar were missing. (b) Three chimpanzees participating in the triadic cooperation task. 


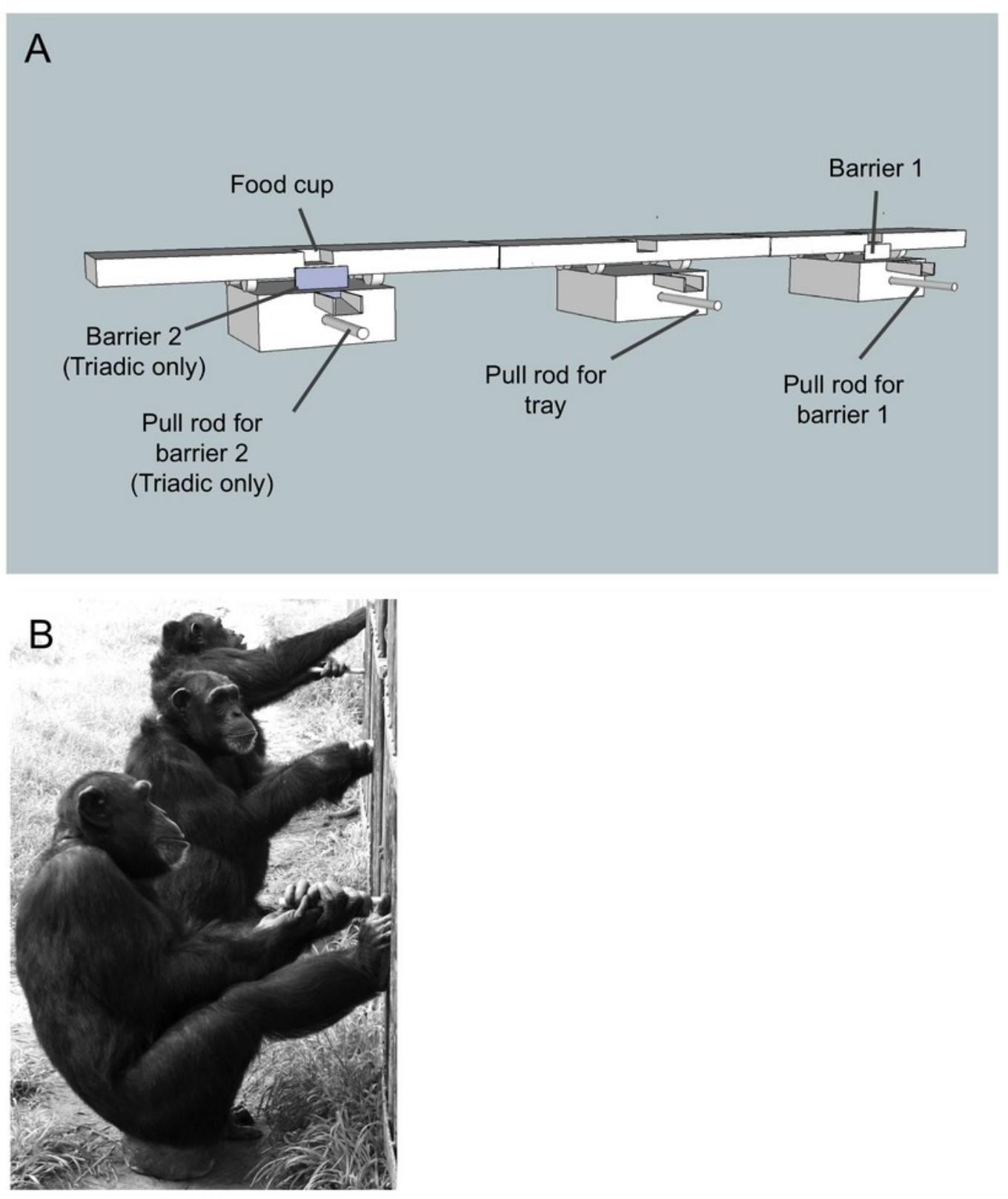




\section{Figure 2}

Affiliation sociogram of the chimpanzee group

Linkages between individuals indicates a significant, positive association. The thicker the line, the stronger the association between individuals. The alpha male (Socko) and alpha female (Georgia) are denoted with an $\alpha$.
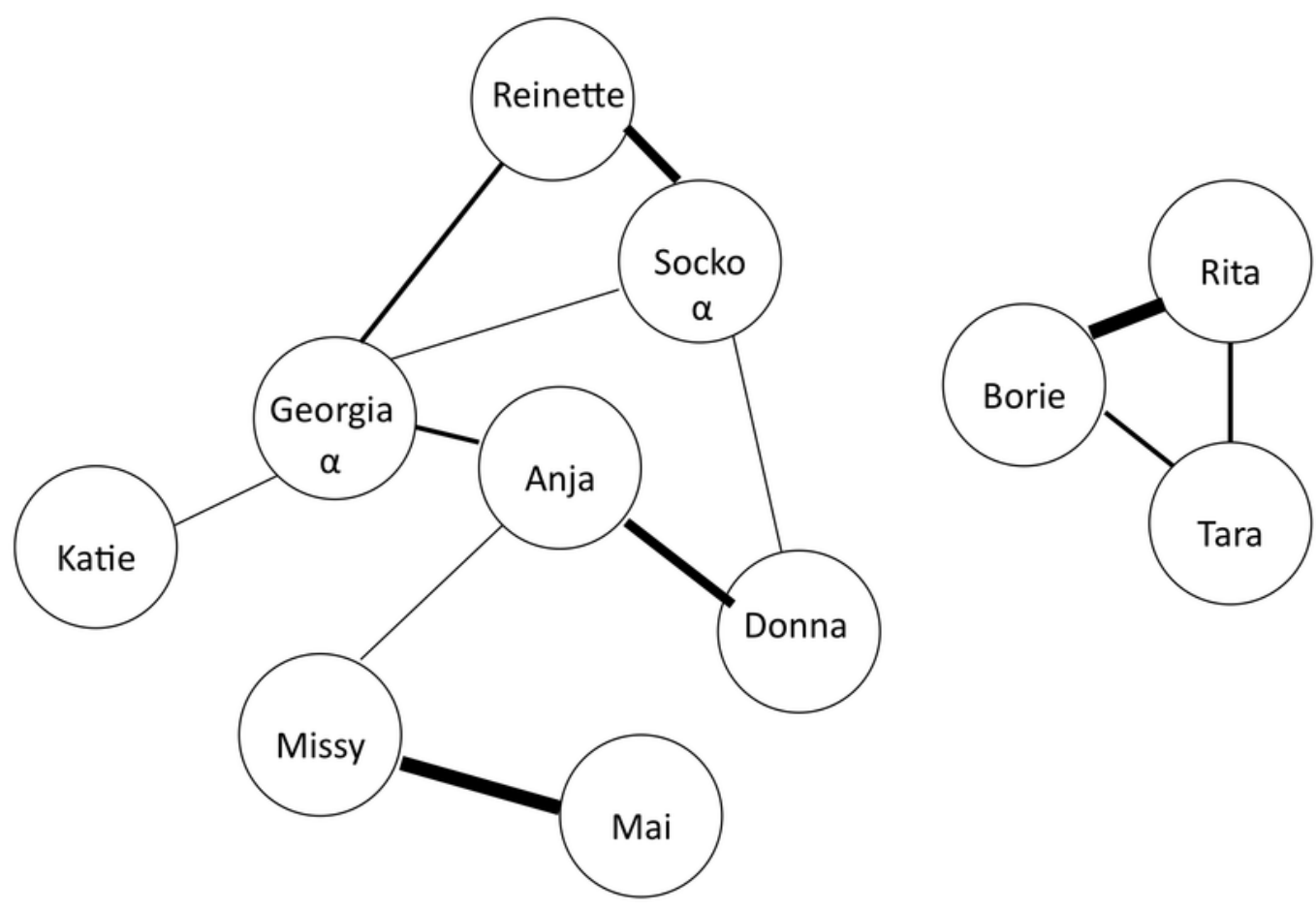

Key:

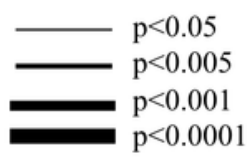




\section{Figure 3}

Success by individual chimpanzee.

The total number of successes for each chimpanzee across all of the test sessions. All individuals except for MA achieved at least 80 successes, with nine chimpanzees achieving over 100.

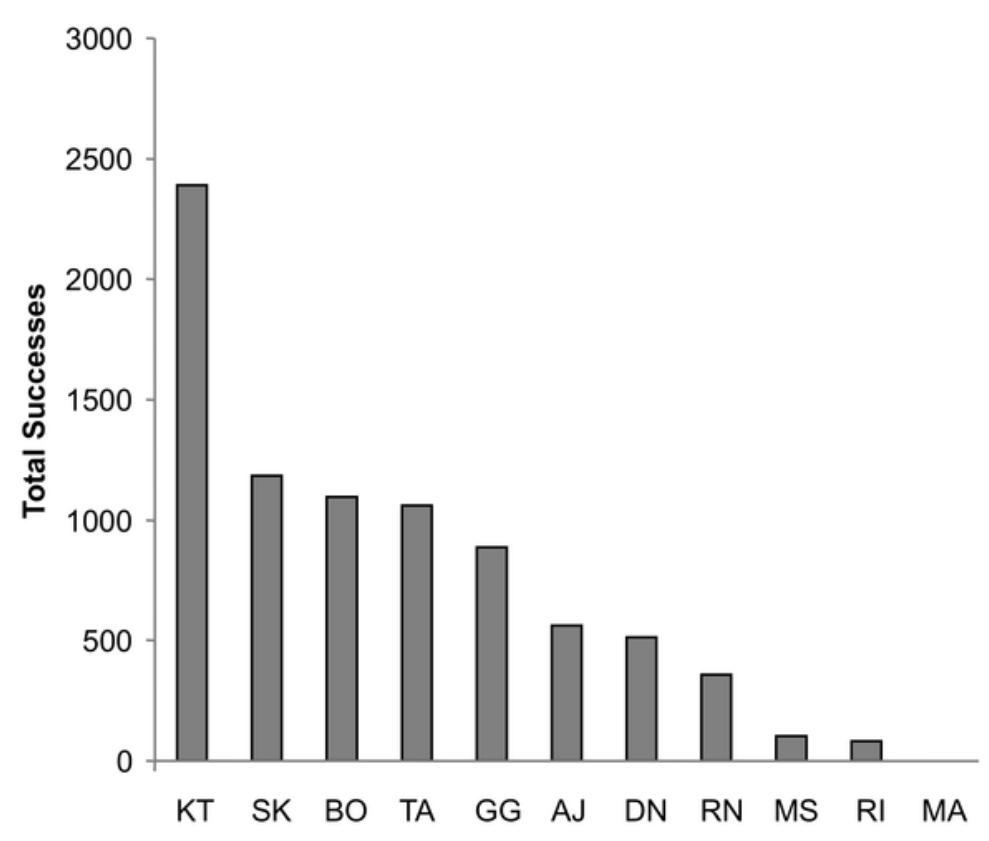




\section{Figure 4}

Number of pulls to success during the acquisition and proficiency phases.

Extraneous pulling that did not lead to success decreased between the two phases for both dyadic and triadic partners. There was no significant difference between the dyadic and triadic conditions.

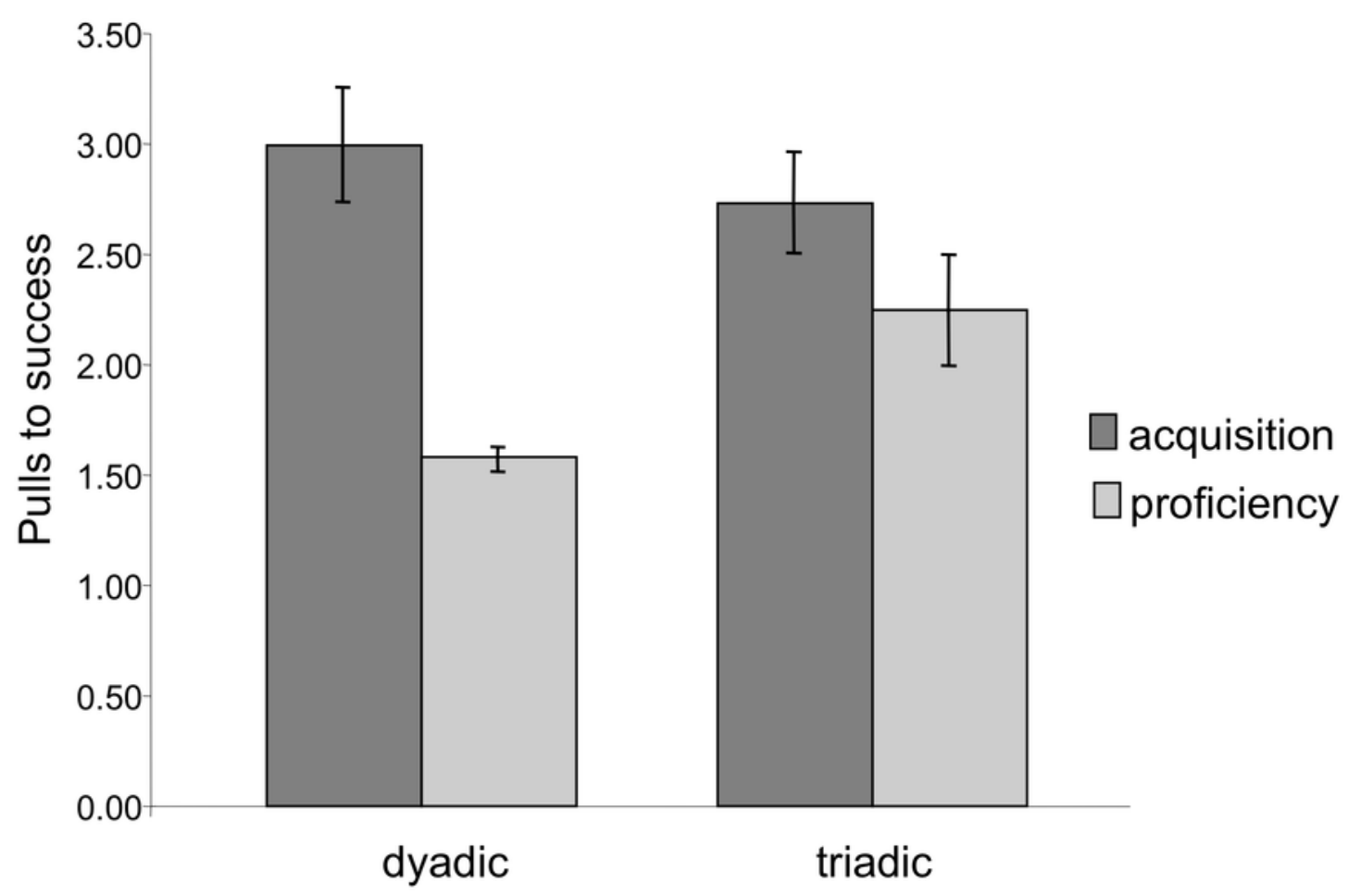




\section{Figure 5}

Comparison of pulling rates per minute during the acquisition and proficiency phases for dyadic cooperation.

Partner "ready" indicates that a partner was both present at the apparatus in a position to be able to pull on the tray. The pattern was similar for triadic pulling rates.

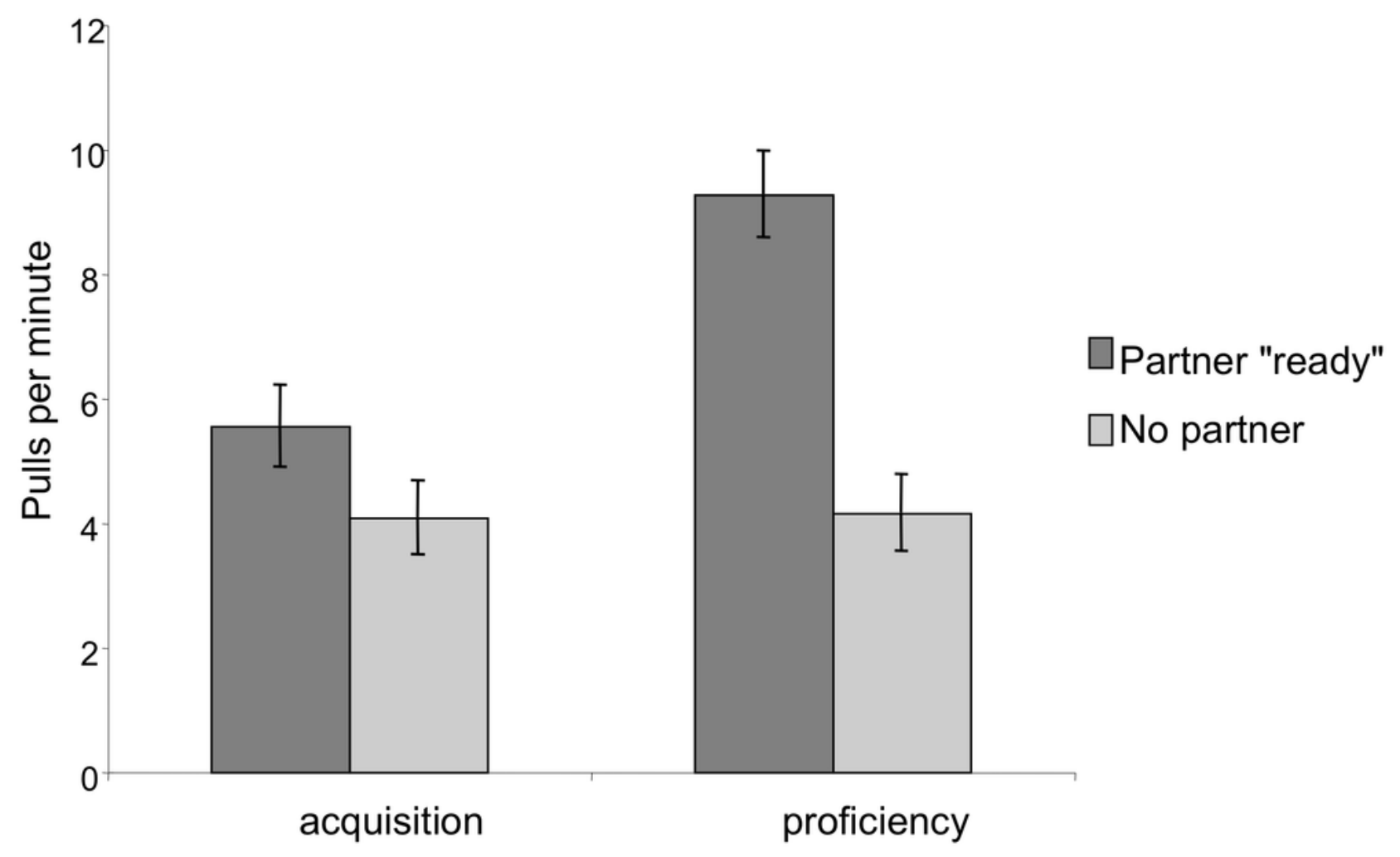




\section{Table 1 (on next page)}

Subjects

Subjects are listed in rank order, with age and any maternal kinship relations provided. Rank was determined using pant grunts, a unidirectional submissive signal. Alpha male and female are denoted with an $\alpha$. 


\begin{tabular}{lcccl}
\hline Chimpanzee & Sex & Age & Rank & Kin \\
\hline SK $\alpha$ & M & 24 & 1 & DN \\
GG $\alpha$ & F & 31 & 2 & BO, RI, KT, TA \\
RN & F & 24 & 3 & \\
BO & F & 47 & 4 & GG, RI, KT, TA \\
MA & F & 47 & 5 & MS \\
KT & F & 22 & 6 & BO, GG, RI, \\
& & & & TA \\
AJ & F & 31 & 7 & \\
RI & F & 24 & 8 & BO, GG, KT, \\
& & & & TA \\
DN & F & 21 & 9 & SK \\
TA & F & 16 & 10 & BO, GG, KT, \\
& & & & RI \\
MS & F & 18 & 11 & MA
\end{tabular}




\section{Table 2 (on next page)}

Results of the best fit GLMM during the proficiency phase

Fixed effects in bold had a significant influence on whether or not an individual approached. In both dyadic and triadic sessions, individuals were more likely to approach others close in rank to themselves, unless the potential partner was kin. 
1

2

\begin{tabular}{lcccc}
\hline Variable & $\mathbf{B}$ & $\mathbf{S E}$ & $\boldsymbol{Z}$ & $\boldsymbol{p}$ \\
\hline Dyadic Proficiency & & & & \\
\hline Fixed Effects & & & & \\
$\quad$ Intercept & -1.98 & 0.46 & -4.30 & $<0.001$ \\
Kin & $\mathbf{- 1 . 3 8}$ & $\mathbf{0 . 6 7}$ & $\mathbf{- 2 . 0 7}$ & $\mathbf{0 . 0 4}$ \\
Affiliation & 0.12 & 0.10 & 1.22 & 0.22 \\
Rank Distance & $\mathbf{- 0 . 3 2}$ & $\mathbf{0 . 0 8}$ & $\mathbf{- 3 . 8 6}$ & $<\mathbf{0 . 0 0 1}$ \\
Kin*rank distance & $\mathbf{0 . 5 2}$ & $\mathbf{0 . 1 3}$ & $\mathbf{3 . 8 0}$ & $<\mathbf{0 . 0 0 1}$ \\
Affiliation*rank distance & -0.03 & 0.03 & -1.12 & 0.26 \\
Random effects & & & & \\
Individual present & variance & 0.26 & & \\
Individual Approaching & variance & 0.23 & & \\
Session & variance & 0.00 & & \\
& & & & \\
\hline Triadic Proficiency & & & & \\
\hline Fixed Effects & & & & \\
$\quad$ Intercept & $\mathbf{- 1 . 7 9}$ & $\mathbf{0 . 3 5}$ & $\mathbf{- 5 . 1 1}$ & $<\mathbf{0 . 0 0 1}$ \\
Kin & -0.47 & 0.45 & -1.05 & 0.30 \\
Affiliation & -0.02 & 0.05 & -.26 & 0.80 \\
Rank Distance & -0.02 & 0.04 & -0.58 & 0.56 \\
Kin*rank distance & $\mathbf{0 . 2 2}$ & $\mathbf{0 . 0 8}$ & $\mathbf{2 . 6 7}$ & $\mathbf{0 . 0 0 7}$ \\
Affiliation*rank distance & 0.02 & 0.01 & 1.26 & 0.21 \\
Random effects & & & & \\
Individual present & variance & 0.21 & & \\
Individual Approaching & variance & 0.19 & & \\
Session & variance & 0.08 & & \\
\hline
\end{tabular}

3 